EUV reflectance characterization of the 94/304 A flight secondary AIA mirror at beamline 6.3.2 of the Advanced Light Source

R. Soufli, E. Spiller, A. L. Aquila, E. M. Gullikson, D. L. Windt

May 24, 2006 
This document was prepared as an account of work sponsored by an agency of the United States Government. Neither the United States Government nor the University of California nor any of their employees, makes any warranty, express or implied, or assumes any legal liability or responsibility for the accuracy, completeness, or usefulness of any information, apparatus, product, or process disclosed, or represents that its use would not infringe privately owned rights. Reference herein to any specific commercial product, process, or service by trade name, trademark, manufacturer, or otherwise, does not necessarily constitute or imply its endorsement, recommendation, or favoring by the United States Government or the University of California. The views and opinions of authors expressed herein do not necessarily state or reflect those of the United States Government or the University of California, and shall not be used for advertising or product endorsement purposes.

This work was performed under the auspices of the U.S. Department of Energy by University of California, Lawrence Livermore National Laboratory under Contract W-7405-Eng-48. 


\title{
EUV reflectance characterization of the 94/304 Å flight secondary AIA mirror at beamline 6.3.2 of the Advanced Light Source
}

\author{
Regina Soufli ${ }^{1}$, Eberhard Spiller ${ }^{1}$, Andy L. Aquila ${ }^{2}$, Eric M. Gullikson ${ }^{2}$, David L. Windt ${ }^{3 *}$ \\ ${ }^{1}$ Lawrence Livermore National Laboratory, Livermore, California \\ ${ }^{2}$ Lawrence Berkeley National Laboratory, Berkeley, California \\ ${ }^{3}$ Columbia Astrophysics Laboratory, New York, New York
}

\begin{abstract}
SUMMARY
The AIA secondary flight mirror, previously coated at Columbia University with $\mathrm{Mg} / \mathrm{SiC}$ for the $303.8 \AA$ channel and $\mathrm{Mo} / \mathrm{Y}$ for the $93.9 \AA$ channel was characterized by means of EUV reflectance measurements at beamline 6.3.2 of the Advanced Light Source (ALS) synchrotron at LBNL on January 10, 2006. Paul Boerner (LMSAL) also participated in these measurements.
\end{abstract}

\section{EXPERIMENTAL SETUP}

Prior to depositing any multilayer coatings, the secondary substrate (Tinsley SN002) was cleaned at LLNL using a custom-designed process. Atomic Force Microscopy (AFM) measurements were also performed at LLNL on the secondary flight substrate prior to multilayer coating, on five locations across the mirror surface, demonstrating an average of $0.17 \mathrm{~nm} \mathrm{rms} \mathrm{of} \mathrm{high-spatial} \mathrm{frequency}$ roughness. The AFM results have been discussed in detail in an earlier report ${ }^{1}$. Multilayer coatings for the $93.9 \AA$ AIA channel $(\mathrm{Mo} / \mathrm{Y})$ and $303.8 \AA$ channel $(\mathrm{SiC} / \mathrm{Mg})$ were deposited at Columbia University, using a deposition system that has been described previously ${ }^{2}$. In this system $50-\mathrm{cm}-\mathrm{long}$ planar cathodes are used. The spinning mirror substrate faces down and the magnetron cathodes sputter up. The individual layer thicknesses are determined by controlling the rotational velocity of the substrate as it passes over each cathode. For the coatings described here, the base pressure in the chamber was less than $2 \times 10^{-7}$ Torr, the sputter gas (Ar) pressure was maintained at 1.6 Torr, and the cathodes were operated in constant power mode: $\mathrm{SiC}$ was deposited at $400 \mathrm{~W}, \mathrm{Mg}$ at $400 \mathrm{~W}$, Mo at $200 \mathrm{~W}$ and $\mathrm{Y}$ at $200 \mathrm{~W}$. The development and optimization of the $\mathrm{Mo} / \mathrm{Y}$ and $\mathrm{SiC} / \mathrm{Mg}$ coatings for AIA has been discussed earlier ${ }^{3}$.

All EUV reflectance measurements presented in this report were measured at beamline 6.3.2. of the ALS synchrotron at LBNL. The characteristics of the beamline and its reflectometer have been described in detail earlier ${ }^{4,5}$. Curved optics of up to $200 \mathrm{~mm}$ in diameter can be mapped in this facility. The reflectometer sample stage shown in Figure 1 allows motion of the optic in 3 dimensions, tilt in 2 dimensions and azimuthal rotation of the sample holder. The detector arm has photodiode, CCD camera, and wire detector mounted and can be rotated $360^{\circ}$ around the axis of the

\footnotetext{
${ }^{*}$ Current affiliation: Reflective X-ray Optics, New York
} 
reflectometer chamber. Once an optic is aligned with the CCD camera, custom-designed software allows the operator to pre-calculate for each surface point a table of all coordinates of the sample stage, and program measurements (EUV wavelength scans in the case of the AIA optics) on multiple locations on the mirror surface without any manual input needed in-between scans. Mirrors are measured in their coating fixtures.

For the measurements in the $94 \AA$ region discussed in this report, a 200 lines $/ \mathrm{mm}$ grating was used in the monochromator, and the monochromator exit slit was set to a width of $50 \mu \mathrm{m}$ thus achieving spectral resolving power $\lambda / \Delta \lambda=1000$. Wavelength $\lambda$ was calibrated based on the Be K absorption edge (111.19 $\AA$ ) with a relative accuracy of $0.011 \% \mathrm{rms}$, and could be determined with $0.007 \%$ repeatability. During the measurements, second harmonic and stray light suppression was achieved with a B filter. For higher-order harmonic suppression, an "order suppressor" consisting of three C mirrors at a grazing incidence angle of $8^{\circ}$ was used in addition to the $\mathrm{B}$ filter. In this manner, a spectral purity of $99.9 \%$ or better was achieved throughout the measurements.

For the measurements in the $304 \AA$ region, a 80 lines $/ \mathrm{mm}$ grating was used in the monochromator, and the monochromator exit slit was set to a width of $50 \mu \mathrm{m}$ thus achieving spectral resolving power $\lambda / \Delta \lambda>1000$. Wavelength $\lambda$ was calibrated based on the $\mathrm{Al} \mathrm{L}_{2,3}$ absorption edge (170.89 $\AA$ ) with a relative accuracy of $0.04 \% \mathrm{rms}$, and could be determined with $0.05 \%$ repeatability. During the measurements, second harmonic and stray light suppression was achieved with a Mg filter. For higher-order harmonic suppression, an "order suppressor" consisting of three $\mathrm{C}$ mirrors at a grazing incidence angle of $20^{\circ}$ was used in addition to the $\mathrm{Mg}$ filter. In this manner, a spectral purity of $99.9 \%$ or better was achieved throughout the measurements.

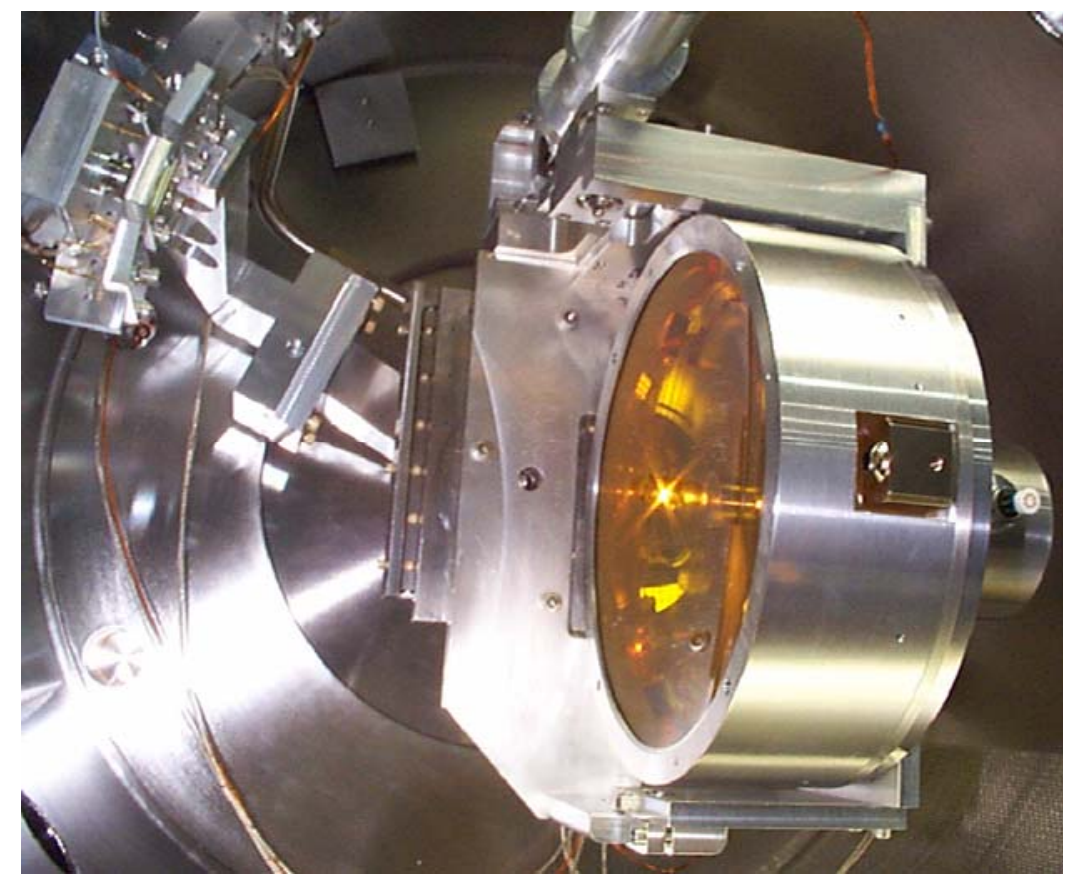

Figure 1: Interior of reflectometer chamber at ALS beamline 6.3.2. with a mounted optic. The detector arm is also shown (top left). 
The ALS storage ring current was used to normalize the signal against the storage ring current decay. The base pressure in the measurement chamber was at $10^{-6}$ Torr. The signal was collected on a Si photodiode detector with a $10 \times 10-\mathrm{mm}^{2}$ active area and acceptance angle of $2.4^{\circ}$. All wavelength and reflectance results were obtained within " $2 \sigma$ " error bars of $0.05 \%$ and $0.4 \%$ relative (the latter limited by photodiode uniformity), respectively. The measurement angle for all flight mirrors and witnesses was set at grazing angle $\theta_{\text {meas }}=87^{\circ}$ and can later be translated to the actual AIA angles of incidence. Geometrical constraints inside the reflectometer prevent measurements at the actual AIA angles of incidence.

\section{RESULTS AND DISCUSSION}

The prescription for the primary and secondary AIA mirror surfaces is given by

$$
z(r)=\frac{1}{R} \cdot \frac{r^{2}}{\left[1+\sqrt{1-(1+K) \cdot(r / R)^{2}}\right]},
$$

where $\mathrm{z}$ is the surface height, $\mathrm{r}$ is the radial position defined from the optical axis of each mirror, $\mathrm{R}$ is the radius of curvature and $\mathrm{K}$ is the conic constant. Table 1 summarizes the values of $\mathrm{R}$ and $\mathrm{K}$, as well as the mirror and clear aperture radii and incidence angles for the AIA mirrors. As is noted in Table 1, the angles of incidence for both mirrors are very close to the normal direction and vary only slightly across the clear aperture. Therefore, uniform multilayer coatings were prescribed for both primary and secondary mirrors of the AIA instrument.

\begin{tabular}{|c|c|c|}
\hline & Primary (concave) & Secondary (convex) \\
\hline R (mm) & $2755.01 \pm 1$ & $1208.57 \pm 0.4$ \\
\hline K & -1.0916 & -5.0766 \\
\hline Mirror outer radius (mm) & 100.0 & 39.9 \\
\hline $\begin{array}{c}\text { Clear aperture } \\
\text { outer radius (mm) }\end{array}$ & 94.0 & 34.0 \\
\hline $\begin{array}{c}\text { Clear aperture } \\
\text { inner radius (mm) }\end{array}$ & 45.1 & 9.0 \\
\hline "Dead strip” width (mm) & 3.05 & 1.0 \\
\hline $\begin{array}{c}\text { Range of incidence angles } \\
\text { within clear aperture }\end{array}$ & $89.1^{\circ}-88.0^{\circ}$ & $90.4^{\circ}-91.8^{\circ}$ \\
\hline
\end{tabular}

Table 1: AIA mirror parameters are shown, including the dead strip width from the center line of each mirror, for the $94 / 304 \AA$ telescope. Angles of incidence are defined from the grazing direction. Optical design by W. A. Podgorski (Smithsonian Astrophysical Observatory). 


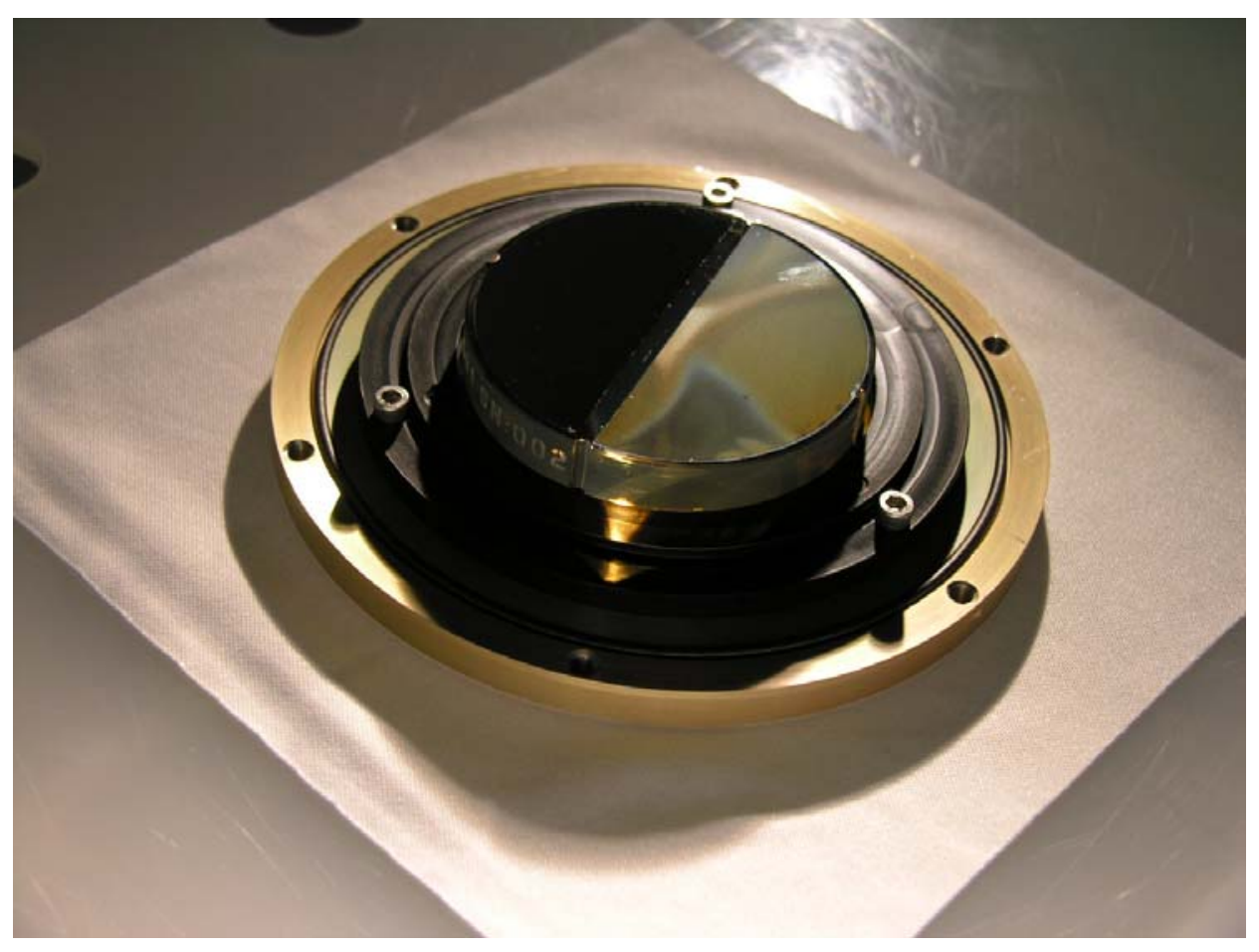

Figure 2: Multilayer-coated AIA flight secondary at $94 \AA$ (left side, $\mathrm{Mo} / \mathrm{Y}$ ) and $304 \AA$ (right side, $\mathrm{SiC} / \mathrm{Mg}$ ) performed at Columbia University. The mirrors are shown on the cleanroom bench inside the LLNL multilayer lab on January 11, 2006, after EUV reflectance measurements were completed at the ALS and prior to installing and packing in the SAO shipping containers.

The main motivation for the EUV reflectance measurements at the ALS was to examine if the areas of discoloration observed on the $303.8 \AA$ multilayer coating, shown in Figure 2, affect in any way the performance of the mirror. 

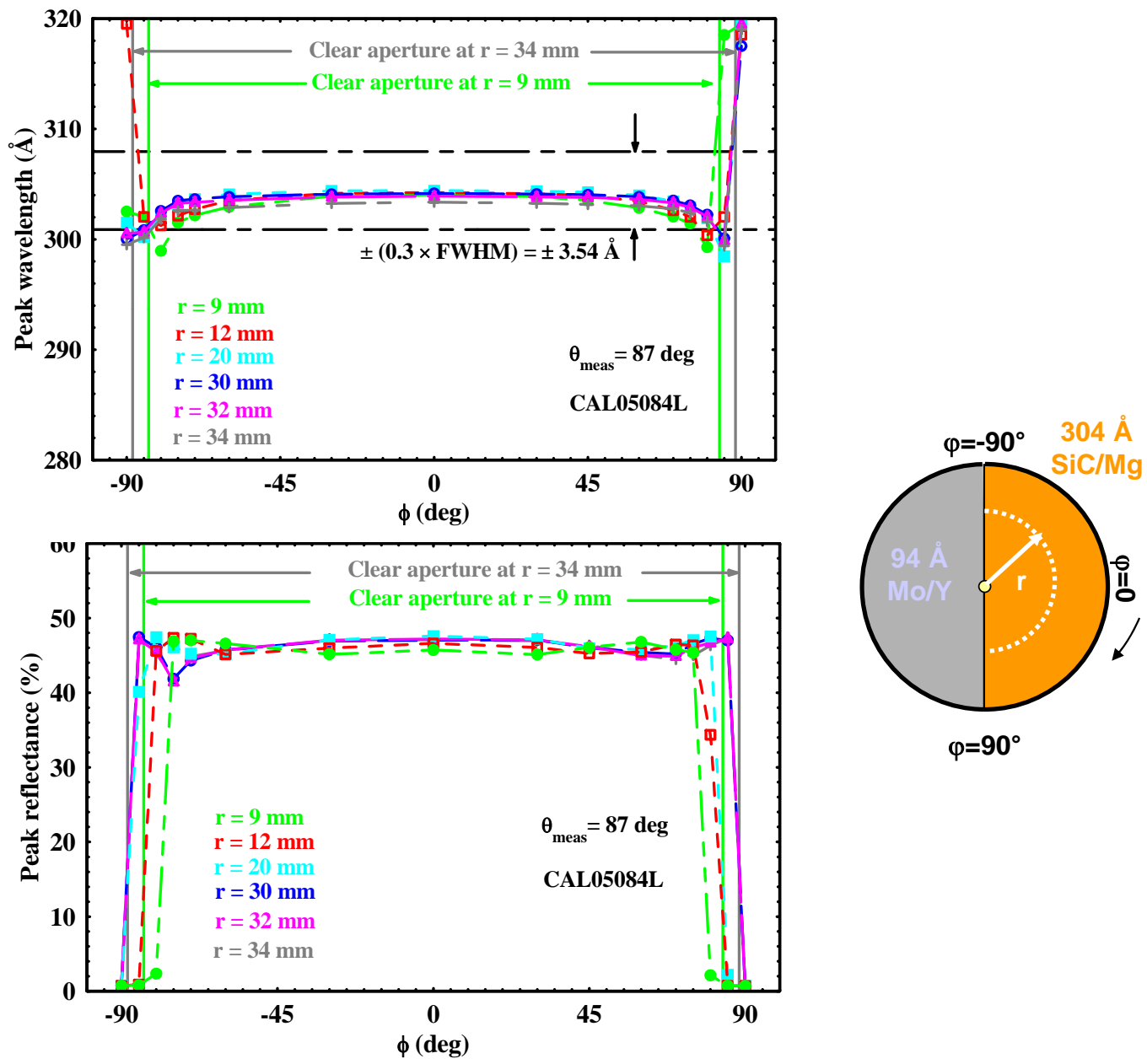

Figure 3: Peak wavelength (top) and reflectance (bottom) results for the $303.8 \AA$ secondary mirror, measured at 96 locations across the surface.

The EUV reflectance of the $303.8 \AA$ secondary coating was measured with 2-dimensional scans on 96 locations across the surface. Six radii $\mathrm{r}=9,12,20,30,32,34 \mathrm{~mm}$ were measured at 16 azimuth angles $\varphi= \pm 90^{\circ}, \pm 85^{\circ}, \pm 80^{\circ}, \pm 75^{\circ}, \pm 70^{\circ}, \pm 60^{\circ},+45^{\circ}, \pm 30^{\circ}, 0^{\circ}$. The azimuth locations were selected to coincide with the areas of discoloration shown in Figure 2, in order to assess the performance of the mirror. The results are plotted in Figure 3. It should be noted that $\mathrm{r}=9,34 \mathrm{~mm}$ are at the edge of the clear aperture and, due to the specified "dead strip" width of $1 \mathrm{~mm}, \varphi= \pm 90^{\circ}$ is outside the clear aperture for all radii and $\varphi= \pm 85^{\circ}$ is outside the clear aperture for $\mathrm{r}=9 \mathrm{~mm}$.

For comparison purposes, the EUV reflectance of the $93.9 \AA$ secondary coating side was also measured with 2-dimensional scans on 78 locations across the surface. Six radii $r=9,12,20,30,32$, $34 \mathrm{~mm}$ were measured at 13 azimuth angles $\varphi= \pm 90^{\circ}, \pm 85^{\circ}, \pm 80^{\circ}, \pm 75^{\circ}, \pm 60^{\circ}, \pm 30^{\circ}, 0^{\circ}$. The results are plotted in Figure 4. 

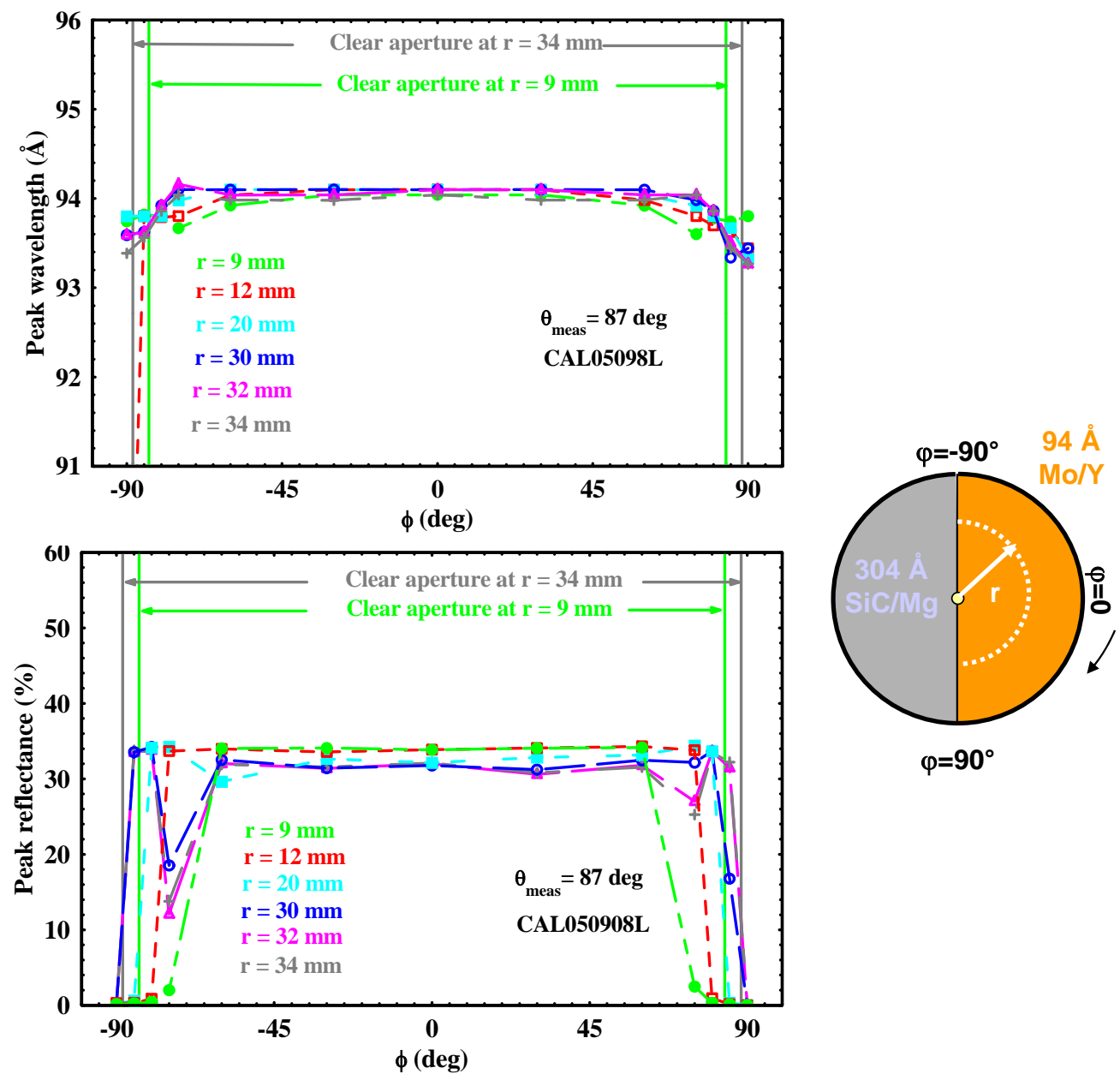

Figure 4: Peak wavelength (top) and reflectance (bottom) results for the $93.9 \AA$ secondary mirror, measured at 78 locations across the surface.

An examination of the EUV reflectance data from the 303.8 A coating (Figure 3, bottom plot) reveals areas of slightly reduced reflectivity in the regions $\varphi<-60^{\circ}, \varphi>60^{\circ}$, which in part overlap with the areas of discoloration shown in Figure 2. Nevertheless, Figure 4 (bottom plot) demonstrates that reflectivity is reduced in exactly the same areas (with respect to the coating fixture and the Dmask) on the $93.9 \AA$ coating. Therefore, a side-by-side comparison of the 93.9 and $303.8 \AA$ reflectance data indicates that the areas of reduced reflectance on both coatings appear to be a result of geometric/shadowing effects that may occur during multilayer deposition and are uncorrelated with the areas of discoloration on the $303.8 \AA$ coating. The reflectance variation of the $93.9 \AA$ side is much more pronounced than the variation of the $303.8 \AA$ side, consistent with a wavelength / film roughness scaling effect which would further support the above argument. The measured peak wavelength of the $303.8 \AA$ coating (Figure 3, top plot) does not demonstrate any variations that could be correlated with the areas of discoloration. 
At Paul Boerner's suggestion, extended wavelength scans were performed on one location of each coating side, to measure the suppression of nearby emission lines: the Fe XVI line (335.4 $\AA$ ) for the $303.8 \AA$ coating and the Ne VIII line $(98.3 \AA)$ for the $93.9 \AA$ coating. The results of these measurements are plotted in Figures 5 and 6, respectively.

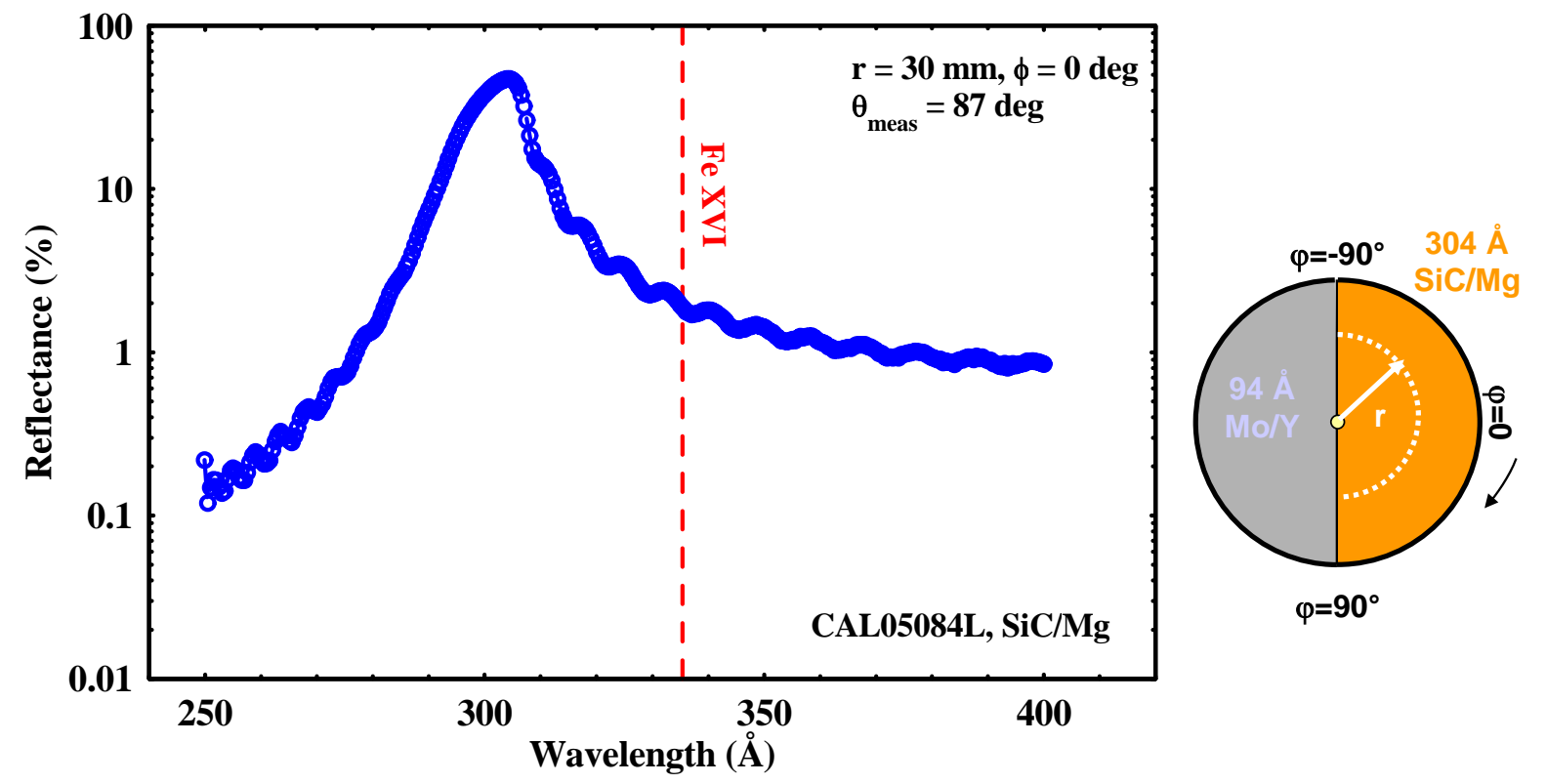

Figure 5: Reflectance vs. wavelength measurement on one location near the center of the clear aperture of the $303.8 \AA$ secondary mirror.

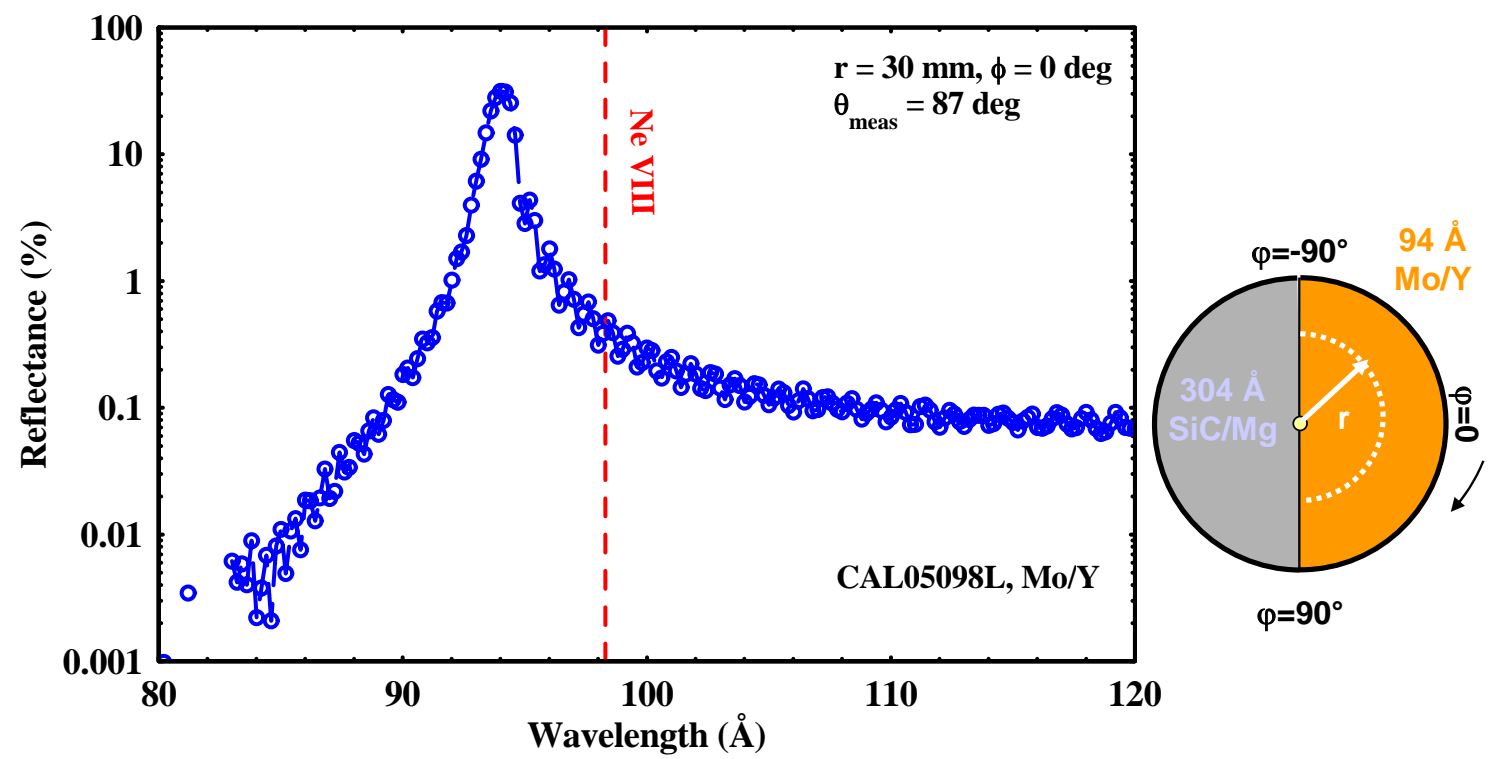

Figure 6: Reflectance vs. wavelength measurement on one location near the center of the clear aperture of the $93.9 \AA$ secondary mirror. 


\section{REFERENCES}

${ }^{1}$ R. Soufli, S. L. Baker and J.C. Robinson, "Atomic Force Microscope (AFM) measurements and analysis on substrates AIA-1001-001, -002 made by Tinsley", report to SAO, August 11, 2005.

${ }^{2}$ D. L. Windt and W. K. Waskiewicz, 'Multilayer facilities for EUV lithography', J. Vac. Sci. Technol. B, 12, 38263832 (1994).

${ }^{3}$ R. Soufli, D. L. Windt, J. C. Robinson, E. A. Spiller, F. J. Dollar, A. L. Aquila, E. M. Gullikson, B.

Kjornrattanawanich, J. F. Seely, L. Golub "Development and testing of EUV multilayer coatings for the atmospheric imaging assembly instrument aboard the Solar Dynamics Observatory", Proc. SPIE 5901, 59010M (2005).

${ }^{4}$ J. H. Underwood, E. M. Gullikson, "High-resolution, high-flux, user friendly VLS beamline at the ALS for the 50-1300 eV energy region," J.Electr. Spectr. Rel. Phenom. 92, 265-272 (1998).

${ }^{5}$ E. M. Gullikson, S. Mrowka, B. B. Kaufmann, "Recent developments in EUV reflectometry at the Advanced Light Source," in Emerging Lithographic Technologies V, E. A. Dobisz eds., Proc. SPIE 4343, 363-373 (2001). 